\title{
Oxygen disorder in ice probed by x-ray Compton scattering
}

\author{
Ch. Bellin, ${ }^{1}$ B. Barbiellini, ${ }^{2}$ S. Klotz, ${ }^{1}$ T. Buslaps,${ }^{3}$ G. Rousse,,${ }^{1}$ Th. Strässle, ${ }^{4}$ and A. Shukla ${ }^{1}$ \\ ${ }^{1}$ Institut de Minéralogie et Physique de la Matière Condensée, Université UPMC, UMR CNRS 7590, case 115, 4 place Jussieu, \\ F-75252 Paris Cedex 05, France \\ ${ }^{2}$ Physics Department, Northeastern University, Boston, Massachusetts 02115, USA \\ ${ }^{3}$ European Synchrotron Radiation Facility (ESRF), Boîte Postale 220, F-38043 Grenoble-Cedex, France \\ ${ }^{4}$ Laboratory for Neutron Scattering, Paul Scherrer Institut, 5232 Villigen, Switzerland
}

(Received 6 July 2010; published 15 March 2011)

\begin{abstract}
We use electron momentum density in ice as a tool to quantify order-disorder transitions by comparing Compton profiles differences of ice VI, VII, VIII, and XII with respect to ice Ih. Quantitative agreement is found between theory and experiment for ice VIII, which is the fully hydrogen ordered phase. Robust signatures of the oxygen disorder are identified in the momentum density for the VIII-VII ice phase transition. The unique aspect of this work is the determination of the fraction $n_{e}$ of electron directly involved in phase transitions as well as the use of position space signatures for quantifying oxygen site disorder.
\end{abstract}

DOI: 10.1103/PhysRevB.83.094117

PACS number(s): 78.70.Ck, 31.70.Ks, 71.15.-m

\section{INTRODUCTION}

Water exhibits a wide range of crystalline and amorphous ices. To date at least 15 crystalline phases and 3 amorphous phases ${ }^{1}$ have been identified. The richness and complexity of the phase diagram of water stems from the hydrogen bond which is solely responsible for the formation of condensed phases $^{2}$ and the related structural versatility of the tetrahedral packing and proton disorder. The intermediate strength of the hydrogen bond permits a large number of possible variations for bonding in terms of angles and bond lengths, as observed for ices obtained at intermediate pressures below $1 \mathrm{GPa}$, i.e., ices II, III, IV, V, VI, IX, and XII. Among these, the greatest distortions in bond angles and lengths are observed for ice VI and can be traced to its peculiar structure. It is composed of two interpenetrating hydrogen-bonded networks, as is the case for ices produced at higher pressures such as ices VII and VIII. In other terms, ice VI carries features common to both ices made at intermediate pressure and ices made at higher pressure (it has a triple point with ices VII and VIII above $1 \mathrm{GPa}$ ). Ice XII (Refs. 3 and 4) exists within the stability domain of ices V and VI and is composed of noninterpenetrating sevenand eight-molecule rings. ${ }^{5}$ The structures of both ice VII and ice VIII are composed of two interpenetrating tetrahedrally bonded networks.

In this paper, we report on Compton scattering measurements of some of the highest mass density ice structures, i.e., ices VI, VII, VIII, and XII as well as ice Ih used only as reference. Apart from ice VIII, all these ice phases are hydrogen disordered with ice VII being the hydrogendisordered form of ice VIII with an additional site-disorder on the oxygen position. This site disorder is not yet fully understood, 7,8 and $a b$ initio calculations have tried to shed some light on the problem. ${ }^{9}$ Our measurements allow us to calculate the fraction of electrons directly involved in the different ice phase transitions. This is done by measuring accurately the number of "displaced" electrons using the change in shape of a measured Compton profile across various phase transitions. It is made possible by the fact that the area of the profile, corresponding to the total number of electrons, remains constant. $\mathrm{x}$-ray Compton scattering thus provides the opportunity to quantitatively study the phase diagram of ice. In particular we provide insight into the role of oxygen disordering in ice VII when compared to ice VIII.

\section{EXPERIMENTAL PROCEDURE AND CALCULATIONS}

The Compton profile within the impulse approximation ${ }^{10}$ is given by

$$
\begin{aligned}
\mathrm{J}(q, \mathbf{e}) & =\int n(\mathbf{p})(\mathbf{p} \cdot \mathbf{e}-q) d \mathbf{p} \\
& =\sum_{\substack{\text { occupied } \\
\text { states }}} \int \chi^{*}(\mathbf{p}) \chi(\mathbf{p}) \delta(\mathbf{p} \cdot \mathbf{e}-q) d \mathbf{p},
\end{aligned}
$$

where $\mathbf{e}$ is the unit vector along the scattering vector $\mathbf{K}, n(\mathbf{p})$ is the electron momentum density, and $\chi(\mathbf{p})$ is the electron wave function in momentum space, i.e., the Fourier transform of the wave function in real space. ${ }^{11,12}$ Throughout the remainder of this paper we shall use atomic units (a.u.), for which $\hbar=m=1$. $\mathrm{x}$-ray Compton scattering has been shown to be of particular importance in studying bonding in water and ice. ${ }^{13-17}$ The technique is complementary to diffraction techniques, which are principally sensitive to ionic positions. Compton scattering is particularly sensitive to valence electron wave functions and to chemical bonding and as a consequence is well suited to the study of hydrogen bonding. The information provided by Compton scattering about the ground-state electron distribution can be directly related to the Fourier transform of the real-space wave functions, so that one can track the changes in the electronic distribution under a variety of thermodynamic conditions and relate them to the changes in bonding and ultimately to the amount of hydrogen bonds in water. ${ }^{17-21}$

The quenched samples were prepared using a ParisEdinburgh pressure cell. For each ice sample, approximately $100 \mathrm{~mm}^{3}$ of distilled deionized water was loaded in the cell. Recovered ice VI was produced by compressing $\mathrm{H}_{2} \mathrm{O}$ to $1.5 \mathrm{GPa}$ followed by cooling to $80 \mathrm{~K}$ and decompressing the sample to ambient pressure. By cooling ice VI and compressing it up to $5 \mathrm{GPa}$ below $95 \mathrm{~K}$, pure ice VII was produced as described in Ref. 6. Recovered ice VI was 
produced by compressing $\mathrm{H}_{2} \mathrm{O}$ to $1.5 \mathrm{GPa}$ followed by cooling to $80 \mathrm{~K}$ and decompressing the sample to ambient pressure.

Ice XII was prepared by compressing $\mathrm{H}_{2} \mathrm{O}$ at $\approx 77 \mathrm{~K}$ to $1.8 \mathrm{GPa}$. It thus corresponds to the second regime of metastability for this ice, as defined in Ref. 4.

All samples of polycrystalline ice (5-mm-diameter spheres) thus obtained were recovered and stored under liquid nitrogen before use. The experiments were carried out using the high energy beamline (Insertion Device 15B) of the European Synchrotron Radiation Facility in Grenoble, France. ${ }^{22}$ Simultaneous in situ x-ray synchrotron diffraction with a MAR image plate setup was used to check for sample purity and avoid alteration. Rietveld refinements using the FullProf suite $^{23}$ were performed to get accurate determinations of the lattice parameters of the samples and to extract the density of each phase. The synchrotron radiation beam was monochromatized to select $86.8 \mathrm{keV}$ photons which were focused on the sample kept inside a temperature cell under vacuum. Cooling was performed using a classical helium displex cryostat which allows the temperature of each measured sample to be maintained below $10 \mathrm{~K}$ during the measurements.

Compton scattering spectra were measured using a germanium multidetector with a scattering angle of approximately $160^{\circ}$. Four vertically aligned elements of the detector have been used. In order to avoid parasite scattering coming from the sample environment, a set of carefully designed slits were mounted before and after the sample, so as to collimate the incident as well as the scattered radiation, and adjusted in order to confine the scattering volume totally within the sample. The resolution function was deduced from the full width at half maximum (FWHM) of the thermal diffuse scattering peak and is equal to 0.45 a.u. Our statistics range from around $6 \times 10^{6}$ counts (ices Ih, VII, and XII for around $4 \mathrm{~h}$ of data acquisition each) to $1.4 \times 10^{7}$ counts (ices VI and VIII for around $7 \mathrm{~h}$ of data acquisition each) in a bin of 0.03 a.u. at the top of the Compton peak. In this bin, the count rate at the top of the Compton peak was around 100 counts per second for each of the pressure points measured. Raw spectra were corrected for background and absorption and converted into momentum scale. Since we are only interested in the single scattering events, the multiple elastic and inelastic scattering contribution (MSC) was calculated for each measured profile by means of Monte Carlo simulations taking into account beam polarization, sample geometry, and density. ${ }^{24}$ The MSC was then subtracted from measured profiles. Since the Compton profile is a projection of the electron momentum density, its integral results in the total number of electrons and provides a convenient normalization. Here profiles have been normalized to the number of electrons in a water molecule. Since many of the systematic errors are subtracted out when one takes the difference between two Compton profiles (CPs), the results are presented in the form of the difference between two profiles, which is thus a very robust quantity.

The program used for the $a b$ initio calculations was CRYSTAL98 (Ref. 25) which is especially suitable for hydrogen-bonded materials. ${ }^{19,26}$ The calculation for ice Ih is described in Ref. 19. For ice VIII, we have taken the structure from Ref. 27. The basis set used to express the orbitals is similar to the one used for ice Ih. ${ }^{19}$ The occupied orbitals used to determine the electron momentum density and the Compton

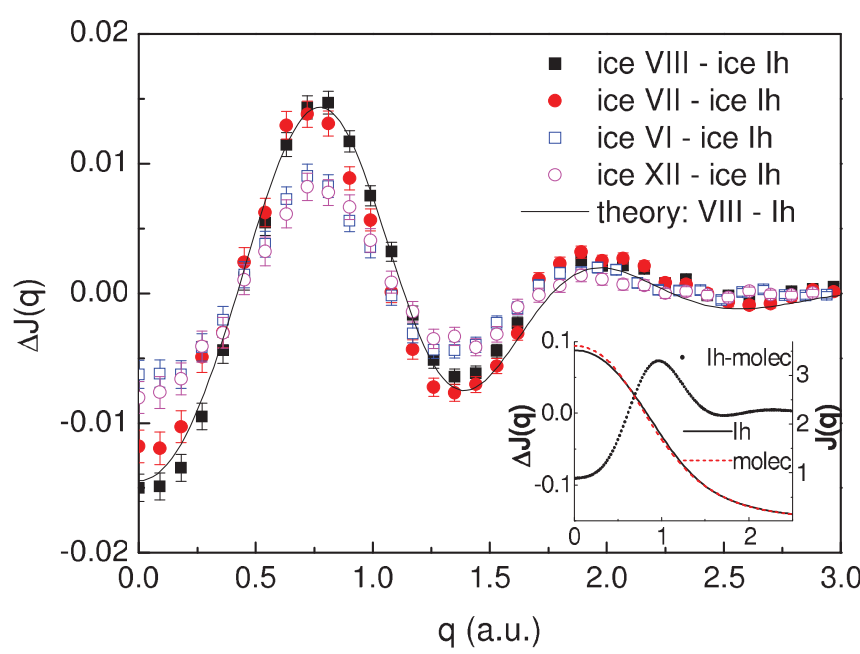

FIG. 1. (Color online) Experimental Compton profile (CP) differences between ice VIII, ice VII, ice VI, ice XII, and ice Ih taken as reference. The theoretical CP difference between ice VIII and ice Ih is also shown. In the inset, calculated isolated water molecule and ice Ih CPs are shown, together with their difference. The water molecule $\mathrm{CP}$ is narrower than the ice Ih $\mathrm{CP}$ showing a higher localization of electronic density in momentum space, i.e., higher delocalization in position space due to the lack of the repulsive antibonding interaction.

profiles were calculated within the restricted Hartree-Fock scheme.

\section{RESULTS AND DISCUSSION}

Figure 1 presents the difference of CPs measured for ices VI, VII, VIII, and XII, using the profile measured for ice Ih as a reference. Two sets of near identical CPs differences can be identified: the first set concerns [ice VIII-ice Ih] and [ice VII-ice Ih] (solid symbols in Fig. 1) and the second set concerns [ice VI-ice Ih] and [ice XII-ice Ih] (open symbols in Fig. 1). The only theoretical CP difference [ice VIII-ice Ih] matches remarkably with the corresponding experimental one. If the amplitudes of the observed wiggles clearly differ between both sets, the shape is the same with coinciding extrema (at $0, \sim 0.75, \sim 1.35$, and $\sim 1.8$ a.u. in each case). All these ice phases have differing crystalline structures but since we use isotropic polycrystalline samples we observe similar oscillatory features representative of local structure, for all phases. In fact, the main contribution to the oscillation is due to an antibonding, repulsive interaction between neighboring water molecules. ${ }^{28-30}$ Other small contributions come from charge polarization ${ }^{31}$ and transfer effects. ${ }^{19}$ In water the amplitude of the oscillation is also related to the coordination but in all ice polymorphs the water molecules are always hydrogen-bonded to four neighbors within an approximately tetrahedral coordination. ${ }^{2}$

Table I summarizes the densities of the studied ices as deduced from our Rietveld refinements together with the averaged apparent $r_{\mathrm{OH}}$ and $r_{\mathrm{OO}}$ lengths, which were taken from the literature sources indicated. The density of the studied ices under similar thermodynamical conditions is the important parameter for the observed amplitudes of the CP differences: the higher the density is with respect to that found in 
TABLE I. Densities and distances $r_{\mathrm{OH}}$ and $r_{\mathrm{OO}}$ for ices Ih, VI, VII, VIII, and XII, ranked in order of decreasing densities. Our ices being recovered, densities are given at ambient pressure and are deduced from Rietveld refinements of the diffraction spectra measured for each of our samples. Values for $r_{\mathrm{OH}}$ and $r_{\mathrm{OO}}$ are taken from references indicated.

\begin{tabular}{|c|c|c|c|c|c|}
\hline & Ice VIII (Ref. 6) & Ice VII (Ref. 6) & Ice VI (Refs. 32,33) & Ice XII (Refs. 3,4, 34) & Ice Ih \\
\hline Density $\left(\mathrm{g} \mathrm{cm}^{-3}\right)$ & 1.50 & 1.50 & 1.32 & 1.29 & 0.92 \\
\hline$r_{\mathrm{OH}}(\AA)$ (mean value) & 0.968 & 0.911 & 0.961 & 0.960 & 0.985 \\
\hline$r_{\mathrm{OO}}(\AA)$ (mean value) & 2.892 & 2.890 & 2.774 & 2.792 & 2.764 \\
\hline
\end{tabular}

ice Ih, the higher are the amplitudes. Ices VIII and VII have a similar density, as do ices VI and XII, and this leads to the two sets of $\mathrm{CP}$ differences identified above. A straightforward explanation stems from the fact that the second moment of the $\mathrm{CP}$ is a measure of the electronic kinetic energy ${ }^{11}$ and thus a broader profile implies a higher electronic kinetic energy. As ice gets denser, the antibonding, repulsive interaction between neighboring water molecules implies more localized orbitals in position space and an increase in the kinetic energy. ${ }^{28}$ This leads to broader CP for ices VI, VII, VIII, and XII compared to ice $\mathrm{Ih}$, as seen by the negative $\mathrm{CP}$ difference at $q=0$ a.u when the ice Ih CP is subtracted.

Regarding the local structural parameters $r_{\mathrm{OH}}$ and $r_{\mathrm{OO}}$ (Table I), both ices VI and XII exhibit very close values, reflected in similar CPs. Ice VII is the fully disordered form of ice VIII, so that both of them have an overall similar structure. Thus, it is not surprising to find similar CPs also for ices VII and VIII. Nevertheless even though $r_{\mathrm{OO}}$ is almost the same for both these ices, the apparent $r_{\mathrm{OH}}$ in ice VII measured by neutron diffraction is 0.911 (Ref. 6), i.e., much smaller than that in ice VIII. This effect comes from oxygen site disorder. The sites are "displaced by $\delta \approx 0.1 \AA$ from the average position," leading to "an apparent bond-length shortening of $0.05 \AA$ " (Ref. 6). This apparent site disorder is not yet fully understood, and a controversy exists between two possible displacements for the oxygen atom along $\langle 111\rangle$ axes $^{7}$ or $\langle 100\rangle$ axes. $^{8}$ Our samples being polycrystalline we cannot pinpoint the directional origin of the disorder. However the oxygen site displacement and the apparent $r_{\mathrm{OH}}$ are very similar, independent of the directional origin $(\langle 111\rangle$ or $\langle 100\rangle)$. As a consequence, if the $r_{\mathrm{OH}}$ value given in Table I are corrected by $0.05 \AA$ as defined above the local parameters of ice VII are close to those of ice VIII. We again conclude that gross similarity in the local structure implies gross similarity in bonding and in the resulting $\mathrm{CP}$.

We now look for finer details in the CP differences so as to go beyond these initial conclusions. For this we use a new set of CP differences, using the ordered ice VIII as a reference. Figure 2 accordingly shows the difference of CPs measured for ice VIII with the CPs of ices VI, VII, and XII.

The two CP differences [ice VIII-ice VI] and [ice VIIIice XII] are similar, as expected from the earlier considerations of density and local environment dominating the bonding and the CPs. Ice VI and ice XII are of near identical density but are less dense than ice VIII; hence their CPs are correspondingly broader leading to a larger negative $\mathrm{CP}$ difference at $q=0$ a.u.
The CP difference between ice VIII and ice VII is worth a closer look. Given the identical densities of the two and their similarity of structure, it should be featureless but is clearly nonzero though of small amplitude (less than $0.01 \%$ of the maximum of the total profile). The CP difference being grossly determined by the averaged local structure, one can infer that the oxygen disorder in ice VII impacts the electronic structure (in the sense of electronic delocalization) sufficiently so as to induce a CP difference. The oxygen disorder in real space is related via the Fourier transform of the electronic wave functions to the momentum space and therefore seems to confirm this inference. We consider the autocorrelation function $B(\mathbf{r})$, which is the Fourier transform of the $\mathrm{CP}$ or the projected momentum density: ${ }^{19}$

$$
B(\mathbf{r})=\int \rho(\mathbf{p}) \exp (-i \mathbf{p} \cdot \mathbf{r}) d^{3} \mathbf{p} .
$$

From the convolution theorem, $B(\mathbf{r})$ is just the autocorrelation of the one-electron wave functions. The effect of isotropic oxygen disorder (i.e., "random" oxygen atom displacement with respect to equilibrium positions by $\delta \approx 0.1 \AA$ ) is simulated by convoluting the $B(\mathbf{r})$ of ice VIII by a Gaussian function with FWHM $=0.1 \AA$ (Ref. 11) and applying the Fourier transform to simulate oxygen-disordered ice VIII. The inset in Fig. 2 shows the $\mathrm{CP}$ difference between ice VIII and a

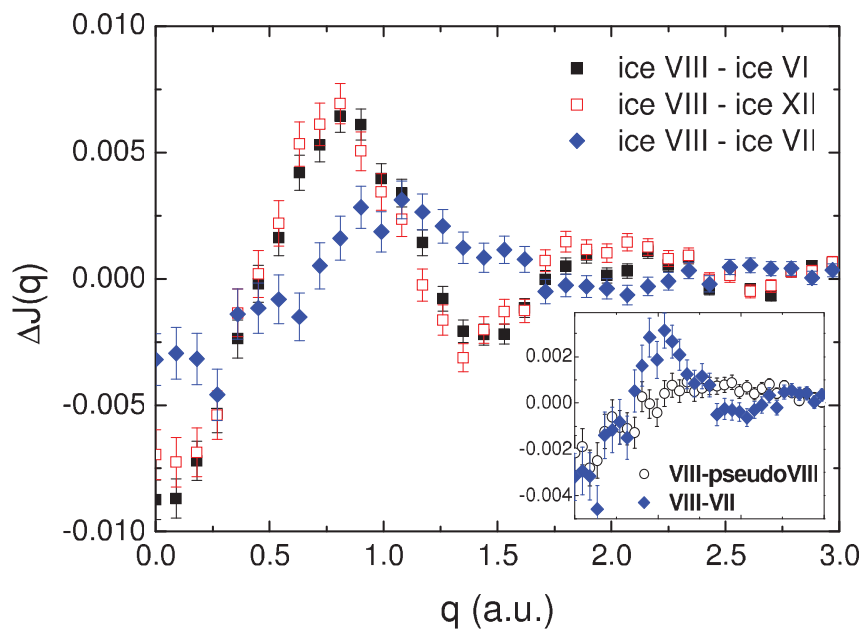

FIG. 2. (Color online) Compton profile differences between ordered ice VIII taken as the reference on the one hand and ice VI, ice XII, and ice VII on the other. The inset shows the difference between ice VIII and a pseudo ice VIII, taking account of oxygen disordering corresponding to an oxygen atom displacement of $0.1 \AA$, compared with the difference ice VIII-ice VII. 
TABLE II. Experimental fraction of electrons $n_{e}$ per molecule obtained for the differences between studied ices and ice Ih taken as reference, together with the $n_{e}$ corresponding to the difference between ice VIII and ice VII. Error bars are based on statistical uncertainty. We checked that the calculated number of displaced electrons remains the same whatever the upper limit of the integration when chosen above 2.8 a.u.

\begin{tabular}{cccccr}
\hline \hline & VIII-Ih & VII-Ih & VI-Ih & XII-Ih & VIII-VII \\
\hline$n_{e}\left(\times 10^{-2}\right)$ experiment & $\mathbf{1 . 4 4} \pm \mathbf{0 . 0 4}$ & $\mathbf{1 . 3 7} \pm \mathbf{0 . 0 5}$ & $\mathbf{0 . 8 1} \pm \mathbf{0 . 0 5}$ & $\mathbf{0 . 7 7} \pm \mathbf{0 . 0 7}$ & $\mathbf{0 . 3 7} \pm \mathbf{0 . 0 4}$ \\
\hline \hline
\end{tabular}

pseudo oxygen-disordered ice VIII. One finds a wide wiggle with the right phase and sign. At low $q$ the $\mathrm{CP}$ difference is negative as in experiment and this finding provides us with a probable explanation of its origin: the oxygen disorder in ice VII.

The effect produced by oxygen disorder can also be illustrated by a comparison between autocorrelation functions of ice VIII and ice VII and calculations for the $\mathrm{H}_{2} \mathrm{O}$ molecule and ice Ih (Fig. 3). The figure shows differences between autocorrelation functions. An extreme case is the $B(\mathbf{r})$ difference between an isolated water molecule calculation (as representative of ultimate disorder) and that for ice VIII or ice Ih. Hydrogen bonding induces a weakening of the covalent bond and changes in the electronic wave functions due to neighboring molecules. This is reflected in the calculated differences where the maximum at short length scales implies a stronger covalent bond in the molecule and the minimum at longer length scales is due to perturbations from hydrogen bonding in the condensed state. The experimental differences between ice VII and ice VIII, though understandably much smaller, show similar behavior with a marked maximum at short length scales and a small minimum at longer length scales. The conclusions are straightforward: oxygen disorder involves a weakening of the hydrogen bonding in ice VII, i.e., a stronger covalent bonding. In other terms, the measured difference between ice VII and ice VIII is due to oxygen site disorder and induces a slightly more molecular nature in the disordered VII phase.

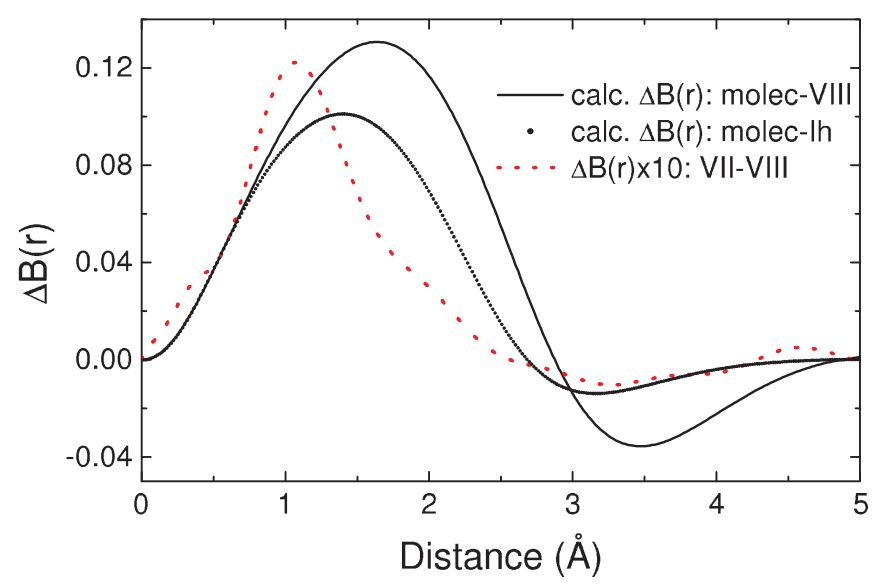

FIG. 3. (Color online) Autocorrelation function differences between calculated water molecule and ice VIII (molec-VIII); calculated water molecule and ice Ih (molec-Ih); and ices VII and VIII (VII-VIII).
Next, we quantify the effect of oxygen disorder. Through the $\mathrm{CP}$ difference it is possible to measure the fraction of electrons directly involved in a phase transition of this kind, that is, the electrons whose wave functions undergo change. The number $n_{e}$ of electrons involved in this change is defined as follows: ${ }^{17}$

$$
n_{e}=\frac{1}{2} \int_{-\infty}^{+\infty}|\Delta J(q)| d q,
$$

where $\Delta J(q)$ is the difference between CPs measured for two different forms of ice. In a previous study, $n_{e}$ allowed us to track specifically the change in the hydrogen bonding coordination in water as a function of the temperature. ${ }^{17}$ Table II presents the experimental quantities $n_{e}$ obtained by means of Eq. (3) for the differences between studied ices and ice Ih taken as reference. The $n_{e}$ corresponding to the difference between ice VIII and ice VII is also shown. The quantity $n_{e}$ relates to change in the local structure of the studied forms of ice and is influenced by at least two different mechanisms, density change and oxygen site disorder. In Table II, we again find the intuitive result mentioned before where denser phases with respect to the reference phase imply higher amplitudes in the CP difference and a higher value for $n_{e}$. This trend shows that disregarding differences in structure, the antibonding interaction serves to increase the electronic kinetic energy as density increases. However in considering the CP difference between ices VII and VIII one remarks that this quantity is unexpectedly large given the near identical values found for VII-Ih and VIII-Ih. This is due to the oxygen disorder which induces a change in the CP different from that induced by a density change, as shown in Fig. 2.

In conclusion, we measure changes in Compton profiles for several phases of ice and trace them in good part to increase in electronic kinetic energy with the density of the phase due to the antibonding, repulsive interaction between neighboring water molecules. We also find a good correlation between the increase in density and the number of displaced electrons for related forms of ice. The anomalous behavior that we find for ice VII arises from the effect of the oxygen site disorder.

\section{ACKNOWLEDGMENTS}

We acknowledge useful discussions of our results with Geneviève Loupias and helpful technical help from Julien Philippe. Bernardo Barbiellini is supported by the US Department of Energy, Office of Science, Basic Energy Sciences Contract DE-FG02-07ER46352 and Contract DEFG02-08ER46540 (CMSN), and benefited from the allocation of supercomputer time at NERSC and Northeastern University's Advanced Scientific Computation Center (ASCC). 
${ }^{1}$ Victor F. Petrenko and Robert W. Whitworth, Physics of Ice (Oxford University Press, Oxford, 2002); see also M. Chaplin, Water Structure and Behavior [http://www.lsbu.ac.uk/water].

${ }^{2}$ D. Eisenberg and W. Kauzmann, The Structure and Properties of Water (Oxford University Press, Oxford, 1969).

${ }^{3}$ C. Lobban, J. L. Finney, and W. F. Kuhs, Nature (London) 391, 268 (1998).

${ }^{4}$ M. M. Koza, H. Schröber, T. Hansen, A. Tolle, and F. Fujara, Phys. Rev. Lett. 84, 4112 (2000); M. Koza, H. Schöber, A. Tolle, F. Fujara, and T. Hansen, Nature (London) 397, 660 (1999).

${ }^{5}$ M. O'Keefe, Nature (London) 392, 879 (1998).

${ }^{6}$ S. Klotz, J. Besson, G. Hamel, R. J. Nelmes, J. S. Loveday, and W. G. Marshall, Nature (London) 398, 681 (1999).

${ }^{7}$ R. J. Nelmes, J. S. Loveday, W. G. Marshall, G. Hamel, J. M. Besson, S. Klotz, Phys. Rev. Lett. 81, 2719 (1998).

${ }^{8}$ C. Knight and S. J. Singer, J. Chem. Phys. A 113, 12433 (2009).

${ }^{9}$ J. L. Kuo and M. L. Klein, J. Phys. Chem. B 108, 19634 (2004).

${ }^{10}$ I. G. Kaplan, B. Barbiellini, and A. Bansil, Phys. Rev. B 68, 235104 (2003).

${ }^{11} X$-Ray Compton Scattering, edited by M. Cooper, P. Mijnarends, N. Shiotani, N. Sakai, and A. Bansil (Oxford University Press, Oxford, 2004).

${ }^{12}$ P. Eisenberger and P. Platzman, Phys. Rev. A 2, 415 (1970).

${ }^{13}$ E. D. Isaacs, A. Shukla, P. M. Platzman, D. R. Hamann, B. Barbiellini, and C. A. Tulk, Phys. Rev. Lett. 82, 600 (1999).

${ }^{14}$ C. Sternemann, S. Huotari, M. Hakala, M. Paulus, M. Volmer, C. Gutt, T. Buslaps, N. Hiraoka, D. D. Klug, K. Hämäläinen, M. Tolan, and J. S. Tse, Phys. Rev. B 73, 195104 (2006).

${ }^{15}$ K. Nygard, M. Hakala, S. Manninen, A. Andrejczuk, M. Itou, Y. Sakurai, L. G. M. Pettersson, and K. Hämäläinen, Phys. Rev. E. 74, 031503 (2006).

${ }^{16}$ K. Nygard, M. Hakala, S. Manninen, M. Itou, Y. Sakurai, and K. Hämälainen, Phys. Rev. Lett. 99, 197401 (2007).

${ }^{17}$ Patrick H.-L. Sit, C. Bellin, B. Barbiellini, D. Testemale, J. L. Hazemann, T. Buslaps, N. Marzari, and A. Shukla, Phys. Rev. B 76, 245413 (2007).
${ }^{18}$ T. K. Ghanty, V. N. Staroverov, P. R. Koren, and E. R. Davidson, J. Am. Chem. Soc. 122, 1210 (2000).

${ }^{19}$ B. Barbiellini and A. Shukla, Phys. Rev. B 66, 235101 (2002).

${ }^{20}$ M. Hakala, S. Huotari, K. Hämäläinen, S. Manninen, Ph. Wernet, A. Nilsson, and L. G. M. Pettersson, Phys. Rev. B 70, 125413 (2004).

${ }^{21}$ M. Hakala, K. Nygard, S. Manninen, S. Huotari, T. Buslaps, A. Nilsson, L. G. M. Pettersson, and K. Hämäläinen, J. Chem. Phys. 125, 084504 (2006).

${ }^{22}$ P. Suortti, T. Buslaps, P. Fajardo, V. Honkimäki, M. Kretzschmer, U. Lienert, J. E. McCarthy, M. Renier, A. Shukla, Th. Tschentscher, and T. Meinnander, J. Synchrotron Rad. 6, 69 (1999).

${ }^{23}$ J. Rodriguez-Carvajal, Physica B 192, 55 (1993)

${ }^{24}$ J. Chomilier, G. Loupias, and J. Felsteiner, Nucl. Instrum. A 235 , 603 (1985).

${ }^{25}$ V. R. Saunders, R. Dovesi, C. Roetti, M. Causà, N. M. Harrison, R. Orlando, and C. M. Zicovich-Wilson, CRYSTAL98 User's Manual (University of Torino, Torino, 1998).

${ }^{26}$ B. Barbiellini, Ch. Bellin, G. Loupias, T. Buslaps, and A. Shukla, Phys. Rev. B 79, 155115 (2009).

${ }^{27}$ K. Umemoto and R. M. Wentzcovitch, Phys. Rev. B 71, 012102 (2005).

${ }^{28}$ T. Ghanty, V. N. Staroverov, P. R. Koren, and R. Davidson, J. Am. Chem. Soc. 122, 1210 (2000).

${ }^{29}$ A. H. Romero, P. L. Silvestrelli, and M. Parrinello, J. Chem. Phys. 115, 115 (2001).

${ }^{30}$ A. Shukla, B. Barbiellini, T. Buslaps, and P. Suortti, Z. Phys. Chem. 215(10), 1315 (2001).

${ }^{31}$ S. Ragot, J.-M. Gillet, and P. J. Becker, Phys. Rev. B 65, 235115 (2002).

${ }^{32}$ B. Kamb, Science 150, 205 (1965).

${ }^{33}$ W. F. Kuhs, J. L. Finney, C. Vettier, and D. V. Bliss, J. Chem. Phys. 81, 3612 (1984).

${ }^{34}$ S. Klotz, G. Hamel, J. S. Loveday, R. J. Nelmes, and M. Guthrie, Z. Kristallogr. 218, 117 (2003). 